Baltic Astronomy, vol.4, 363-377, 1995.

\title{
THE AM CVn TYPE STARS: AN OVERVIEW
}

\author{
J.-E. Solheim
}

Institutt for Matematiske Realfag, Universitet i Troms $\varnothing, N-9037$ Troms $\varnothing$, Norway

Received September 1, 1995.

Abstract. A review of observations and theoretical developments for the AMCVn type stars since the previous workshop is given. For AMCVn itself, line profile variations are interpreted as a sign of an elliptical disk in a permanent superoutburst state. This object also shows "flares" and UV-wind profiles. A new family member, EC 15330-14, with almost identical properties as AMCVn, is discovered. For GP Com some doubt has been raised on its group membership, but this question is not settled.

Key words: stars: cataclysmic variables - stars: individual: AM CVn, GP Com, CR Boo.

\section{Introduction}

In this overview, I will concentrate on new observations and analysis of the AM CVn stars since the last workshop. Recent general overviews are found in the forthcoming book about the cataclysmic variables (Warner 1995a) and in a review paper from the same author (Warner 1995b). Warner has introduced the name AM CVn stars for what we used to call interacting binary white dwarf systems. This is in accordance with the naming practice in astronomy, where a particular family of stars is named after the first one identified with the family characteristics.

Warner (1995a) uses model calculations by Savonije et al. (1986), who concludes that during the binary evolution the mass losing secondary enters a phase where the thermal time scale becomes much longer than the gravitational time scale. The result is that the secondary star becomes semi-degenerate instead of degenerate as we used to believe before. This changes the period vs. mass 
relation, so we must accept heavier secondaries and primaries for the same $q=M_{1} / M_{2}$ values.

Since we met last time Judi Provencal has finished her thesis with title "The Interacting Binary White Dwarf Systems" (Provencal 1994), and she has published a paper about AM CVn which she calls "The unusual helium variable AMCVn" (Provencal et al. 1995). She will also soon publish official WET paper from XCOV1 on CR Boo. The official paper from XCOV4 on AMCVn will be sent to the co-authors for comments after this workshop (Solheim et al. 1996).

The most interesting developments concerning AMCVn since the last workshop are: the discovery of absorption line profile modulations with a period of $13.4 \mathrm{~h}$ by Patterson et al. (1993), the identification of some of the AMCVn stars as superhumpers by Warner (1995b) and magnitude variations of about $0.5 \mathrm{mag}$ for AMCVn which I will describe later.

We must also announce a new family member, published by D. O'Donoghue et al. (1994). This has the name EC 15330-1403, and it is almost a clone of AMCVn, with nearly the same spectrum and fundamental frequency. The temporal spectrum is simpler - it has fewer peaks and harmonics than AMCVn, and the fundamental frequency is present, not missing as in AMCVn. With this addition the AMCVn family has grown from 5 to 6 members and we can do statistics...

However, not everything is good in the family. The membership of GP Com has been challenged (Ulla et al. 1996) since it has some notable spectral differences, and a new period of $4.868 \mathrm{~h}$ have been detected, while the old period of $46.52 \mathrm{~min}$ is not confirmed. Based on this Ulla et al. (1996) claim that this system has an orbital period of $4.868 \mathrm{~h}$ and cannot be an AMCVn object. However, ROSAT has detected modulations with the previous orbital period of 46.52 minutes, so the matter is not settled.

Low signal-to-noise, high resolution IUE spectra have been obtained for three of the family members, but only the results for AM CVn are published (Solheim \& Sion 1994). IUE time, awarded for observing the high state of V803 Cen and CR Boo, could not be used since they did not go into the high state as predicted. In the discovery paper (Wood et al. 1987) it was shown that CR Boo spent about 80 per cent of its time in the high state. During a watch in 1993 and 94 it almost never was found in the high state. And if it was the high state, it went down immediately instead of staying for weeks as it did in the old days. The conclusion is that something 
has happened with $\mathrm{CRBoo}$. We should monitor it more closely to find out.

In Fig. 1 results from XCOV1 is shown. CR Boo was then monitored during a campaign of 16 days long and we can identify three phases, one stable high state, one stable low state and a number of shorter outbursts, not quite making it to a stable high level. We identify the short outbursts as normal ones and the long outburst as a superoutburst, and we notice immediately that the outburst have a much shorter rise and fall time than is observed for cataclysmic variables (CV) with the normal hydrogen disk. (Cannizzo 1996). This can give us important clues for the study of helium disk properties.

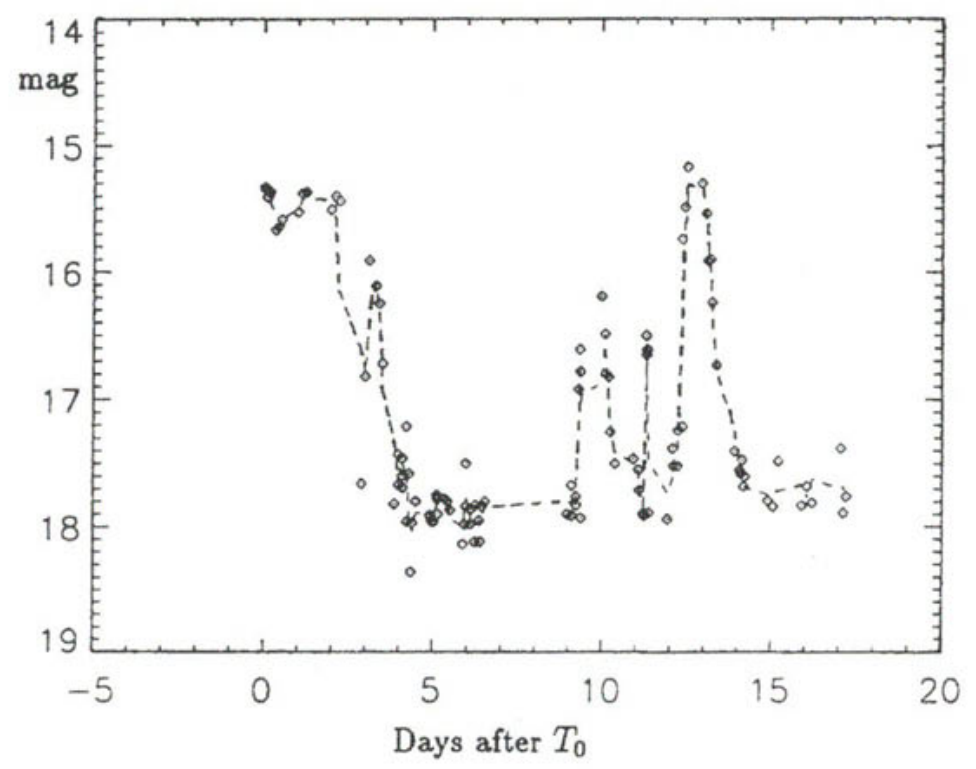

Fig. 1. The light curve for CR Boo, averaged over $3 \mathrm{~h}$, during XCOV1. The magnitudes are in the WET system.

Fig. 1 shows magnitudes in the WET system, which is defined by the passband of detector + atmosphere. What it means depend on the object studied and the calibration stars used. If we in the future shall use the WET system for variable AMCVn stars, it is necessary to perform a better calibration, and, if possible, to observe in multiple colors. For the outbursting AMCVn objects, it is important to find out if these changes are color related i.e. if there is a delay in $U$ with respect to the $V$, which is observed for ordinary 
CVs. Hint of such a delay is seen in the IUE spectra, but not conclusive (Solheim 1993). Fig. 2 shows the result of monitoring CR Boo with the Carlsberg Automatic Transit Circle (CAMC) in 1993 (Argyle 1994). This confirms that CR Boo has changed it properties since the time of discovery. It definitely does not spend 80 per cent of its time in the high state any more.

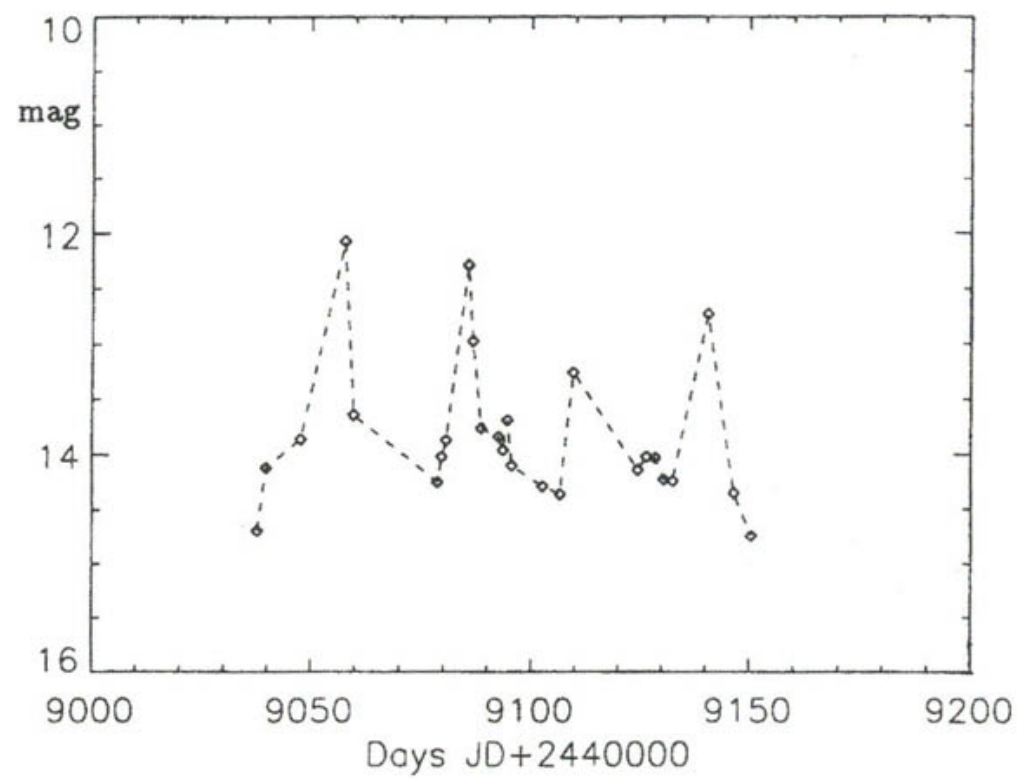

Fig. 2. Monitoring of the $V$ magnitude of CR Boo with the CAMC at La Palma in 1993.

In the following I will describe some new results and give a summary of the tidal instability theory, which I think is an important step to understand the disks and outbursts mechanisms of these objects. I will also give a summary of the ROSAT observations done so far, which identifies some very hot sources in some of the systems. Finally I will present some priorities for future XCOVs on these objects.

During this workshop we will hear more about the secondary object in the AMCVn system by Tanja Nymark (Nymark \& Solheim 1995), light curve modulations in various colors by Christophe Massacand (Massacand \& Solheim 1995) and a simulation of the disk structure by Wood and Simpson (1995) in blasting colors. The controversy between interpreting AMCVn as a pulsator or having 
a tideally variable disk, will be brought to court by the two contestants, Clemens (1995) and O'Donoghue (1995), and the readers must judge who wins the case.

During the next interworkshop period, we plan to do more work on IUE data in Troms $\varnothing$ and obtain at least one sequence of AMCVn spectra taken during cycle 5 of the HST. A WET campaign on the exiting new family member EC $15530-1403$ is planned in 1997 . We also look forward to exiting new results from making disk structure models and atmospheres, and maybe also more knowledge about the accreting objects and the mass donors will come in the near future.

\section{AMCVn variability: it varies more than single white dwarfs do!}

During 1992 and 1993, we managed to get three of the AMCVn stars monitored by the CAMC at La Palma (Argyle 1993, 1994). This system is set up for astrometric observations, but a few variable stars are accepted into the program. In addition they produce magnitudes, well calibrated with a network of standards, accurate to $0.03 \mathrm{mag}$. The limiting magnitude is about 15 . The result for 1992 was not spectacular: V803 was never seen (usually too faint), CR Boo never went brighter than $15 \mathrm{mag}$, and AM CVn was between $V=14.1$ and $14.2 \mathrm{mag}$. It has always been since its discovery in 1962, a low amplitude variable star (Smak 1967). Since V803 Cen was unobservable, it was taken out of the program in 1993. But luckily, CR Boo and AMCVn were kept in the program. The result for AMCVn in 1993 is shown in Fig. 2. It shows that for 5 out of 8 observations, the star was brighter than its usual range, up to 0.5 mag brighter on two occasions.

These observations have three profound consequences:

(1). It will be very difficult to argue that AMCVn is a pulsating single star which does not vary more than the pulsations allow it to do.

(2). The magnitude variations may be a repetition of "flares" on AM CVn, earlier reported by Elsworth et al. (1982) and Marar et al. (1988).

(3). If $\mathrm{AMCVn}$ is a superoutburster, this may mean that it has some super-super-outburst episodes.

Interpretations are widely open: it may have enhanced instabilities in the disk, runaway processes on the accreting star or genuine flares on the secondary. Maybe this is a precursor for a violent event, 
such as a supernova eruption. The conclusion is obvious: AMCVn should be watched and its magnitude calibrated!

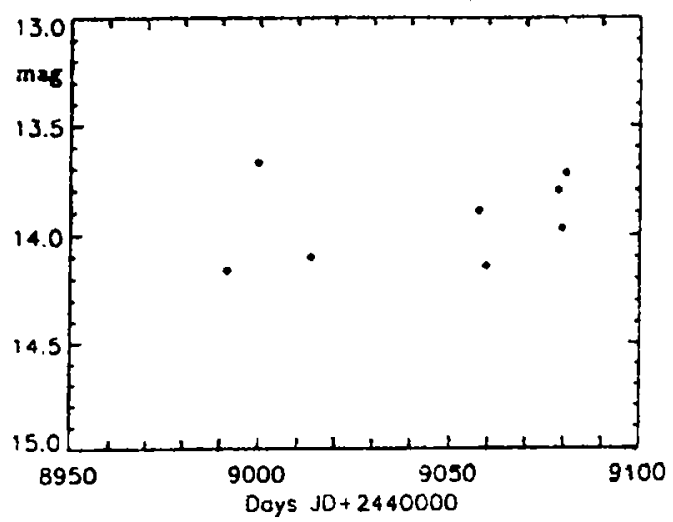

Fig. 3. Monitoring of the magnitudes of AMCVn with the CAMC at La Palma in 1993. The error of observations approximately is of the size of the symbols (Argyle 1994).

\section{Evolution and statistics.}

The AMCVn stars are a result of an unusual evolutionary sequence. The close binary system is a result of an earlier common envelope phase which results in loss of the outer layers by a stellar wind, or recurrent supernova or dwarf supernova outburst. This evolutionary scenario from (Iben \& Tutukov 1991) is shown in Fig. 4. What is left is the carbon core remnant of the primary, and the helium core remnant of an unevolved secondary. Since the starting mass of both components are (5-8) $M_{\odot}$, the clue to their evolution is that mass-loss has been initiated during helium shell flashes, which has effectively stopped helium core burning.

Independently of the starting binary configuration, the evolution is governed by the mass transfer rate and the loss of angular momentum due to gravitational radiation. In the early stages mass transfer is dominating, and the orbit shrinks to one with period of $\sim 10$ min (Iben \& Tutukov 1991, Savonije et al. 1986). Thereafter a long lasting phase of quiet evolution takes place while the orbit is expanding and the secondary looses mass at a decreasing rate. Fig. 5 shows a simplified version of Iben \& Tutukov (1991) evolutionary tracks. A goal for our research in Troms $\varnothing$ during the next few years is to determine the position of the AMCVn objects as precisely as 


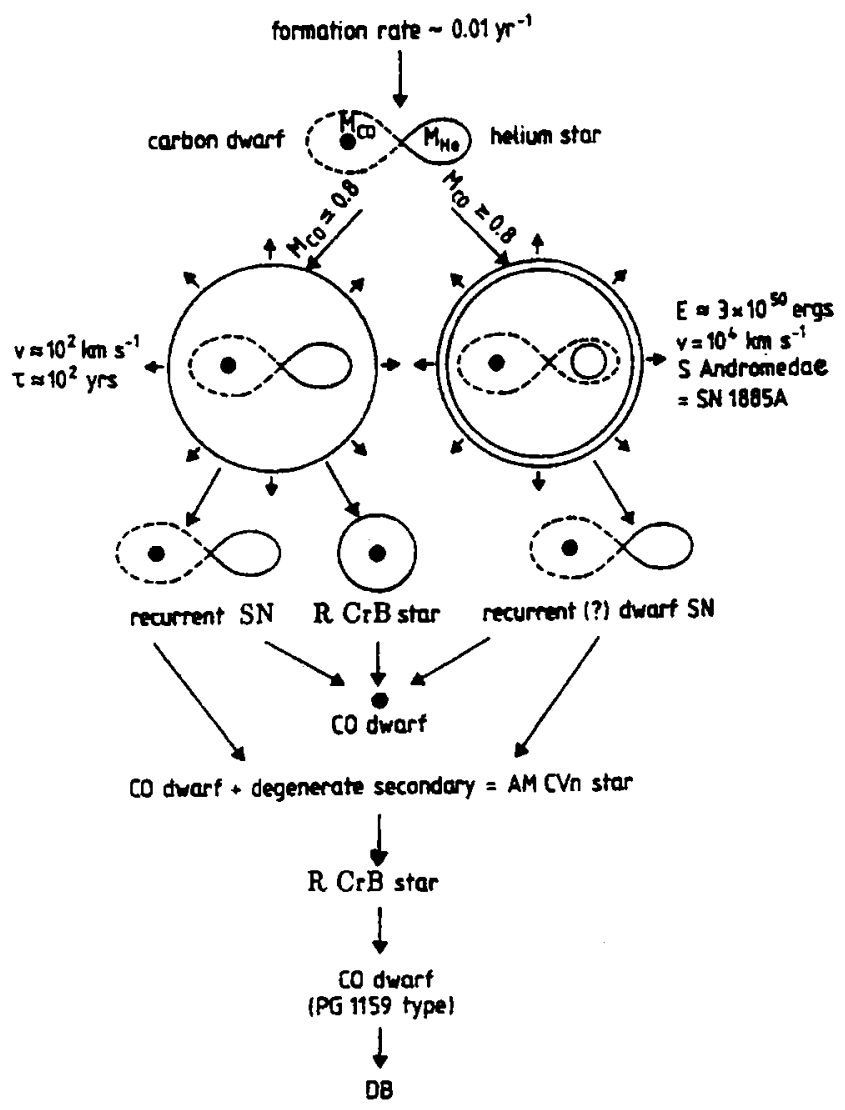

Fig. 4. Evolution of precursors of the helium cataclysmic variables or the AM CVn stars. From Iben \& Tutukov (1991) with some additions.

possible with respect to evolutionary tracks in such a graph, and also to investigate by abundance studies clues to the nature of the precursors. In Fig. 5 three tracks are shown which may lead to systems with helium secondaries.

What happens after the AMCVn stage? From other contributions at this workshop we have heard that the binary channel is the third and maybe the only working channel for making DBs. Since the AMCVn family now has grown to 6 members, and 5 of these are believed to be closer than $100 \mathrm{pc}$, and maybe constitute a complete sample within this distance, Warner (1995b) has done some statistics. He started with the birth rate of AMCVn systems as given in Fig. 4, the space density and the life time of these stars and end 


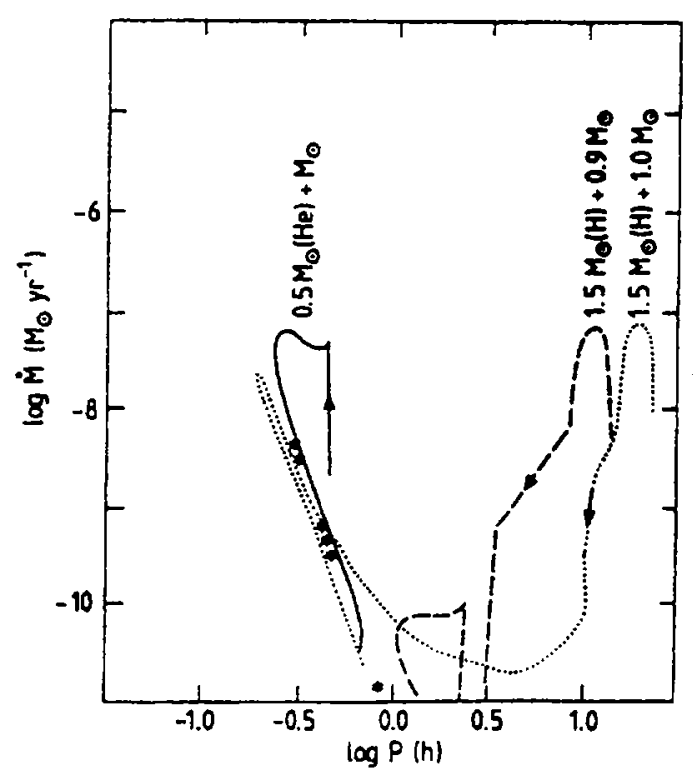

Fig. 5. Evolutionary tracks in the mass transfer vs. period diagram showing the evolution of systems which may end up as the AMCVn stars. Adapted from Iben \& Tutukov (1991). The star symbols marks the expected positions of the AMCVn stars if they are assumed to have a semidegenerate secondary.

up with the space density of descendants $\rho_{\text {dec }}=1 \times 10^{-} 3$, which corresponds to the space density of DB white dwarfs.

In order to prove this connection, we should look for DBs with unusual mass or composition, or even better, look for the hydrogen deficient hot DOs of abnormal mass. Werner (1995) has given us one example in the extremely hot pre-white-dwarf $\mathrm{H} 1504+65$, which has its $\mathrm{H}$ and $\mathrm{He}$ envelope stripped off and a temperature of $170000 \mathrm{~K}$.

There is a flaw in this evolutionary scenario: the mass transfer in late stages of the AMCVn stars becomes so slow that it takes more than the lifetime of the Universe to create a single star. So we must look for pre-white-dwarfs with tiny companions.

One spectacular evolutionary possibility is that the secondary has a small carbon core buried under helium layers, which at some stage in the mass transfer process are stripped away, so carbon will be transferred. This will immediately create much higher opacities in the disk and on the accreting primary, and an expanding carbon rich atmosphere may be created. This can be the birth of a $\mathrm{R} \mathrm{CrB}$ 
object, which later may evolve into a PG 1159-03 object and finally to a DO or DB white dwarf.

\section{The tidal instability model and the AMCVn stars}

It is well known that accretion disks can have thermal limit cycles, where the disk alternates between a high state (outburst) with a radiative disk structure and a low state with an optically thin disk. The reason for this is that the disk temperature, caused by the mass flow rate from the secondary, is within the limits for creating a zone of partial ionization in the disk. This can be explained with the famous $S$ curve in the relation between disk density and the mass transfer rate $\dot{M}$, which is shown in Fig. 6 (Meyer-Hoffmeister \& Ritter 1991). For helium disks this instability works when the temperature is high enough to ionize helium, i.e. $12500 \mathrm{~K}$ (Smak 1983).

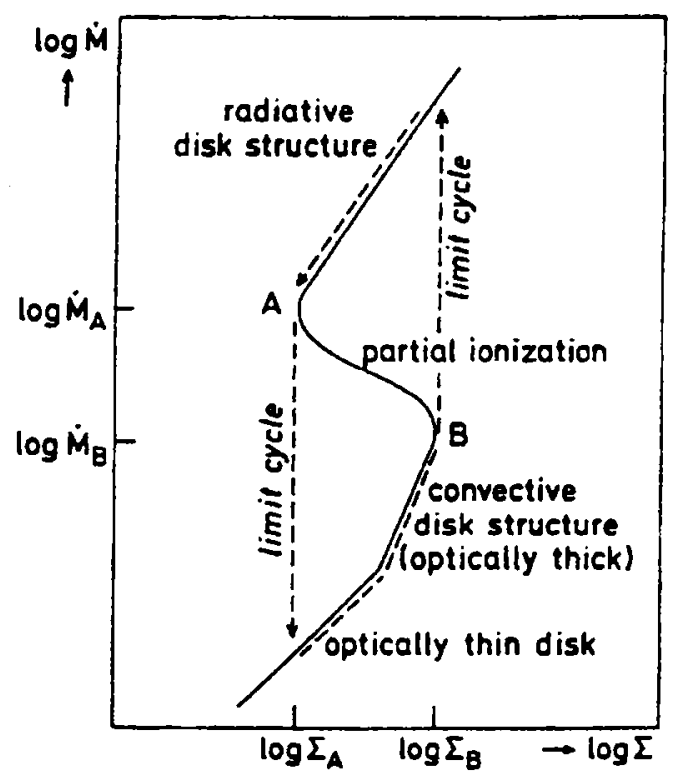

Fig. 6. Scheme of the mass transfer rate versus density relation for accretion disks. The limit cycle between hot and cool states is indicated. From Meyer-Hoffmeister \& Ritter (1993)

For binary systems with small mass ratios and sufficiently high mass transfer rates, simulation shows that if the disk expands beyond 
the $3: 1$ resonance radius for between particle periods in the disk and the binary orbital period, the disk will expand into a tidally driven precessing elliptical structure. The expansion into such a high state can occur during a normal outburst, if enough angular momentum is stored in the disk. We will then get a superoutburst, which lasts longer than normal outbursts and in the light curve we may observe superhump periods which are by a few per cent longer than the orbital period. A schematic presentation of the tidal relaxation cycle is shown in Fig. 7.

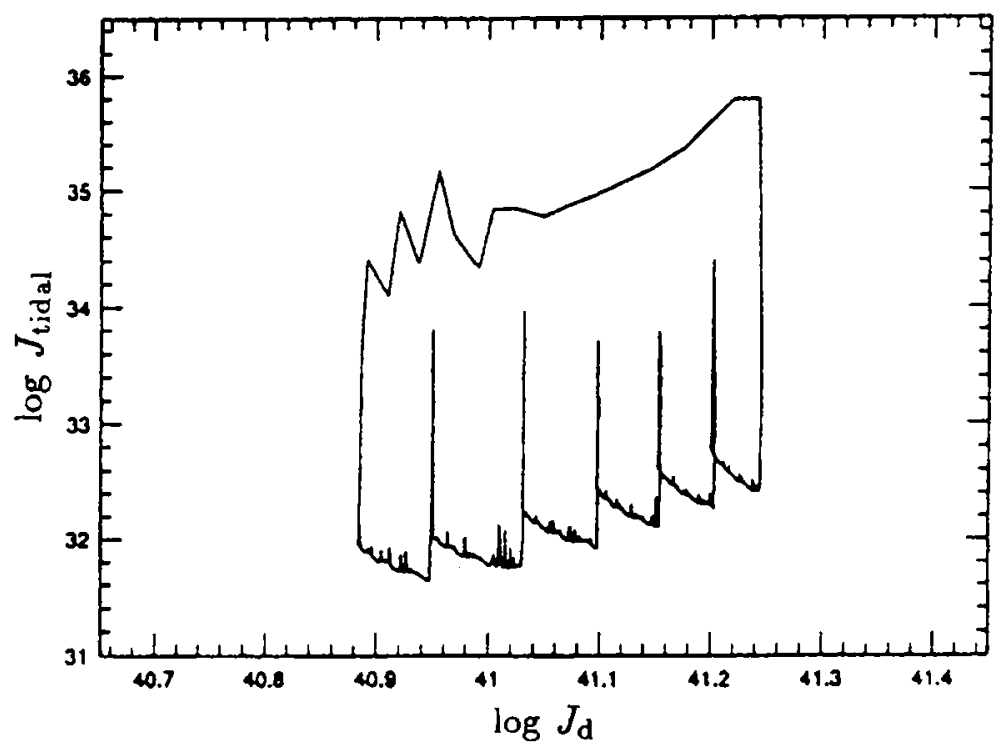

Fig. 7. The tidal relaxation oscillation cycle for the total angular momentum of a disk for a complete super-cycle. The abscissa shows the total angular momentum of the disk and the ordinate is the tidal torque. The spikes represent normal outbursts which are not strong enough to make the disk expansion beyond the tidal instability limit (after Ichikawa et al. 1993).

For the AMCVn stars the discovery of disk precession in AM CVn (Patterson et al. 1993) and the interpretation of superhump and orbital periods a few per cent apart in the WET data for the same object and for CR Boo (Warner 1995a, Solheim 1996), has opened a new avenue for understanding these objects. It seems that AMCVn and maybe EC 15330-14 are permanent superoutbursters, 
which show permanent superhump periods. This is possible if the mass transfer rate is high enough to keep the disk permanently in a high state.

Fig. 8 shows two examples of calculations from Osaki (1995) for the mass transfer rate just below and above the critical mass transfer rate for the hydrogen CVs. If the mass transfer rate is below the critical value, we will observe normal outbursts between superoutbursts. This may be the case for CR Boo as shown in Fig. 1. If the mass transfer rate is just above the critical rate, a series of normal outbursts will make the disk grow into the tidal instability region and stay in a superoutburst state until the mass transfer rate decreases below the critical value. For AMCVn and EC 15330-140, this theory predicts that they will be in the superoutburst state until the period has slowed to $1350 \mathrm{~s}$, what may take 200000 years with the present observed $\dot{P}$.
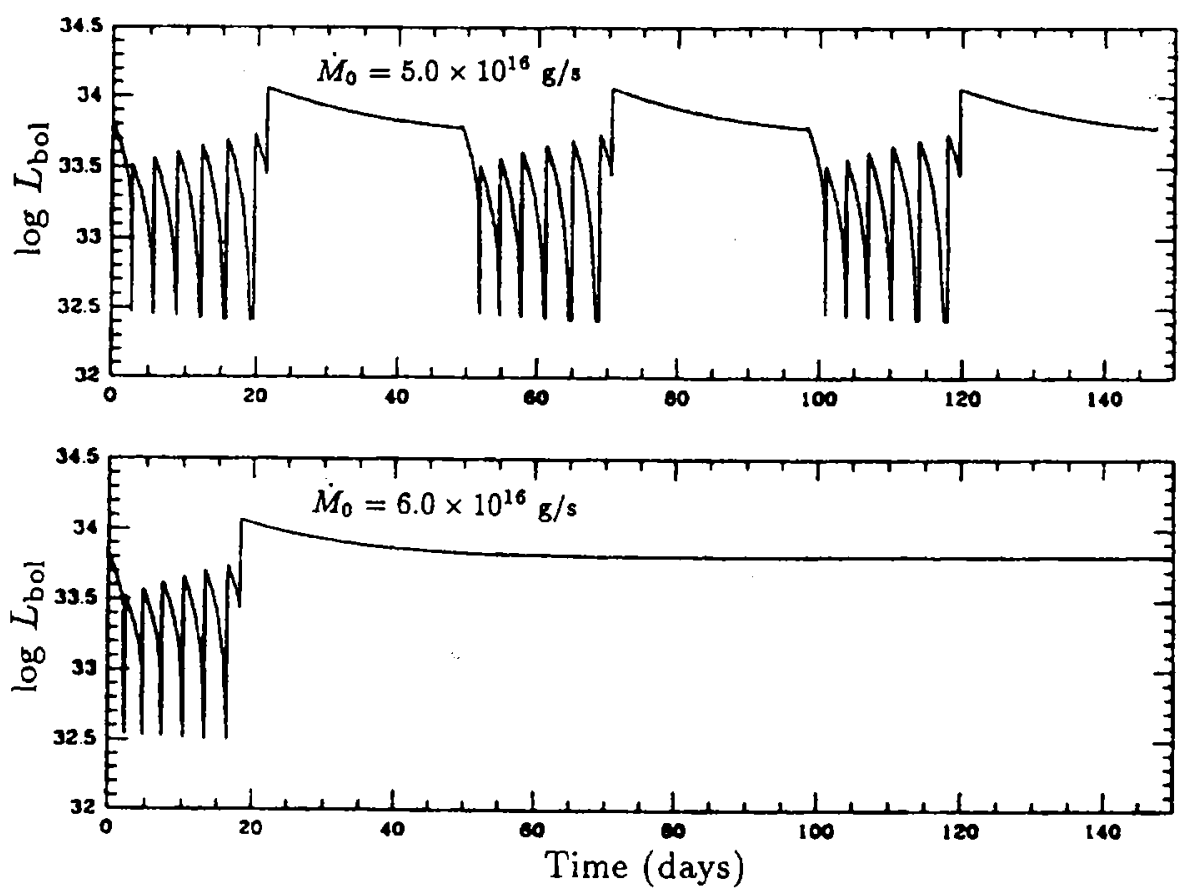

Fig. 8. Bolometric light curves for a mass transferring system with the mass transfer rate just below the critical rate (upper panel) and for a system which is transferring mass at the critical rate (lower panel) (from Osaki 1995). When the critical mass transfer rate is reached, the light curve shows a permanent high state. 
Considering the observed periods, we can divide the AMCVn stars into three groups having analogs among the normal CVs (with orbital periods in parenthesis):

A. High state, permanent superoutbursts; analogs are the nova-like variables AMCVn (17.1 min) and EC $15330(18.6 \mathrm{~min})$,

B. Intermediate state, frequent or intermittent superoutbursts; analogs are the VY Scl type stars: CR Boo (24.5 min), V 803 Cen (26.9 min), CP Eri (28.7 min),

C. Low state, superoutbursts have not been observed yet; analog is the SU UMa type star GP Com (49.5 min).

\section{New spectral information: IUE and ROSAT}

From a high resolution IUE spectrum, Solheim and Sion (1994) have found a $\mathrm{He} \lambda 1640 \AA$ absorption line profile which looks identical to a similar line profile for the coldest DO star HZ 21. In both cases the profiles are blueshifted with an emission peak at about -300 $\mathrm{km} / \mathrm{s}$. This indicates a connection between AMCVn or rather the central star in the AMCVn system and a DO star. It may also tell us that this DO star has an expanding hot atmosphere. Maybe this DO is an AMCVn descendant?

In Troms $\varnothing$ S. Bard (1995a, 1995b) has struggled with stellar atmosphere models, trying to fit a DB spectrum to the AMCVn spectrum from 110 to $1000 \mathrm{~nm}$. No acceptable fit was possible. Then he constructed a disk atmosphere consisting of stellar atmospheres corresponding to the disk effective temperature distribution. This did fit well in the visual part of the spectrum, but not in the far UV, where a source with temperature of $300000 \mathrm{~K}$ had to be added. This could be the central white dwarf with a hot boundary layer, or hot outer atmosphere because of the accretion.

In the ROSAT all sky survey (Beuermann \& Thomas, 1993) with the PSPC counter at $0.07-2.4 \mathrm{keV}$ down to detection limit of 0.04 $\mathrm{c} / \mathrm{s}$, three members of the AMCVn family were detected: GP Com, CRBoo and V803 Cen. They are all members of the groups B or C objects, as described above, and were most likely detected in their low state, with optical thin disks and strong high energy radiation from the accretion region on the central star.

For GP Com, the variability was detected, and pointed observations revealed a two component spectrum with a hot $(2 \mathrm{keV})$ and a less hot $(143 \mathrm{eV})$ source present (Beuermann \& Thomas 1993; van Teeseling \& Verbunt 1994). Both flares, with an increase of hardness 
ratio by a factor of 10 , and spectral variability with the orbital period of $46.52 \mathrm{~min}$ were detected. The modulation can be explained by absorption in rotating matter in the accretion disk, and the flaring is most likely a result of the accreting process. In addition, UV flares have been detected for this object, lasting $1 / 2 \mathrm{~h}$ and increasing the continuum level by $30 \%$. It is not known if the UV and X-ray flares are related.

For AMCVn the pointed observations were done in December 1992. The source was detected at a level of $0.035 \mathrm{c} / \mathrm{s}$, just below the detection limit for the all sky survey. The spectrum showed also two components: one component with extremely hard bremstrahlung of a few $\mathrm{keV}$ temperature and other one, which could be of the size of a white dwarf with a temperature of $86000 \mathrm{~K}$ or $157000 \mathrm{~K}$, depending on the absorption column (van Teeseling 1995). A source with the temperature of $300000 \mathrm{~K}$ can be fitted to the observed spectrum, only if it is much smaller than a white dwarf. Circumbinary matter, detected in the IUE spectrum (Solheim \& Sion 1994), may explain the absorption in this case, but this has to be checked by forthcoming HST observations.

\section{Future WET tasks}

A new member, EC 15330-140, which is more typical AMCVn star than AMCVn, itself should be the highest priority for a WET campaign. Possible multiplets due to beat between superhump and orbital period should be looked for, just as in the case of AMCVn. The spacing will be $14.8 \mu \mathrm{Hz}$ or less, which require at least 3 days to be resolved.

The next highest priority for a normal WET campaign should be $\mathrm{V} 803 \mathrm{Cen}$. It was the second priority of XCOV1, and got a very poor coverage, not more than could be done from Sutherland Observatory alone. A full campaign of at least two weeks should be done in this case, and at least part of it should be done in multicolor mode to get more information about the behavior of the outburst cycles.

The AMCVn stars are also good candidates for campaigns with the three star technique (Breger 1993). The challenge is to detect and verify the period of precession predicted from the elliptical disk models described above. The predictions for precession period modulations are: $A M C V n$ : 13.4h; EC 15330-14: >18.7 h; CR Boo: $1.2 \mathrm{~d}$; V 803 Cen: $>2$ d. 
A long three-star or hybrid technique (Breger \& Handler 1993) campaign on the two last objects will also give good data for studying the outburst cycles.

Finally, all the members of the AMCVn family have erratic behavior and should be monitored regularly. There are $\dot{P}$ questions still pending, and the outburst cycles need to be monitored more closely. Even flares on AMCVn and EC 15330-14 may occur.

On the theoretical side, apart of the mentioned questions, concerning the disk model, one outstanding problem remains: what happens with the temporal spectrum of a DB star when it accretes?

Acknowledgments. I am especially thankful to the Carlsberg Meridian Circle group at La Palma for including AMCVn and CR Boo into their program, and to the Norwegian Research Council for providing travel funds for my participation in the Ames workshop.

\section{References}

Argyle R. W. 1993, Carlsberg Meridian Catalogue La Palma, Cophenhagen University Observatory, Royal Greenwich Observatory and Real Instituto y Observatorio de la Armada en San Fernando, vol. 7

Argyle R. W. 1994, Carlsberg Meridian Catalogue La Palma, Cophenhagen University Observatory, Royal Greenwich Observatory and Real Instituto y Observatorio de la Armada en San Fernando, vol. 8

Bard S. 1995, University of Troms $\varnothing$, Institutt for Matematiske Realfag, Master Thesis

Bard S. 1996, in CVs and Related Objects (IAU Colloq. No. 158), eds. A. Evans \& J.H. Wood, in press

Beuermann K. , Thomas H.-C. 1993, Adv. Space Research, 13 (12), 115 Breger M. 1993, in Stellar Photometry - Current Techniques and Future Developments (IAU Colloq. No. 136), eds. C. S. Butler \& I. Elliott, Cambridge Univ. Press, p. 106.

Breger M, Handler G. 1993, Baltic Astronomy, 2, 468

Cannizzo J. 1996, in CVs and Related Objects (IAU Colloq. No. 158), eds. A. Evans \& J.H. Wood, in press

Clemens C. 1995, Baltic Astronomy, 4, 142

Elsworth W., Grimshaw L., James J.F. 1982, MNRAS, 201, 45

Iben I. Jr., Tutukov A.V. 1991, ApJ, 370, 615

Ichikawa S., Hirose M., Osaki Y. 1993, PASJ, 45, 243

Marar T.M.K. et al. 1988, A\&A, 189, 119

Massacancd C., Solheim J.-E. 1995, Baltic Astronomy, 4, 378 
Meyer-Hoffmeister E., Ritter H. 1993, in The Realm of Interacting Binary Stars, eds. J. Sahade, G.E. McCluskey \& Y. Kondo, Kluwer Academic Publishers, p. 143

Nymark T.K., Solheim J.-E. 1995, Baltic Astronomy, 4, 386

O'Donoghue D. et al. 1994, MNRAS, 271, 910

O'Donoghue D. 1995, Baltic Astronomy, 4, 413

Osaki Y. 1995, PASJ, 47, L11

Patterson J., Halperen J., Shambrook A. 1993, ApJ, 419, 83

Provencal J.L. 1994, University of Texas, Ph.D. Thesis

Provencal J.L. et al. 1995, ApJ, 445, 927

Savonije G.J., de Kool M., van den Heuvel E.P.J. 1986, A\&A, 155, 51

Smak J. 1967, Acta Astron., 17, 255

Smak J. 1983, Acta Astron., 33, 333

Solheim J.-E. 1993, in White Dwarfs: Advances in Observation and Theory, ed. M. Barstow, Kluwer Academic Publishers, p. 395

Solheim J.-E. 1996, in CVs and Related Objects (IAU Colloq. No. 158), eds. A. Evans \& J.H. Wood, in press

Solheim J.-E., Sion E. 1994, A\&A, 287, 503

Solheim J.-E. et al. 1996, in preparation

Solheim J.-E. 1996, in the 2nd International Colloquium of Hydrogen Deficient Stars, ed. C.S. Jeffery, in press

van Teeseling A., Verbunt F. 1994, A\&A, 292, 519

van Teeseling A. 1995, private communication

Ulla A. 1996, in the 2nd International Colloquium of Hydrogen Deficient Stars, ed. C.S. Jeffery, in press

Warner B. 1995a in Cataclysmic Variable Stars, Cambridge University Press, in press

Warner B. 1995b, Ap\&SS 225, 249

Werner K. 1995, Baltic Astronomy, 4, 340

Wood M.A., Winget D.E., Nather R.E., Hessman F.V., Liebert J., Kurtz D.W., Wesemael F., Wegner G. 1987, ApJ, 313, 757 